\title{
NEUMOMEDIASTINO ESPONTANEO: ENFISEMA RETROFARINGEO FORMA DE PRESENTACION NO HABITUAL
}

\author{
Drs. Pilar Ovalle $B^{(1)}$, José $D$. Arce $V^{(2)}$.
}

1. Facultad de Medicina: Universidad de los Andes.

2. Servicio de Radiología. Clínica Santa María.

\begin{abstract}
Spontaneous pneumomediastinum is a rare disorder, usually benign and self-limited. It occurs in young people without an apparent cause or precipitating factor. Cervical symptoms exclusive, without subcutaneous emphysema are infrequent. We presente two cases that were evaluated in emergency room and the diagnosis was erroneous. The initial study included a neck and cervical $X$-ray film. Second lecture in radiology department detected prevertebral cervical air and the patients were posteriorly studied with neck and thorax CT and pneumomediastinum was confirmed. None pulmonary disorder was found. Pneumomediastinum phisiopathologhy and also aggressive clinic investigation in these patients are discussed.
\end{abstract}

Key words: Cervical pain, Pneumomediastinum, Spontaneous pneumomediastinum.

Resumen: El neumomediastino espontáneo, es una patología generalmente benigna y autolimitada, caracterizado por la presencia de aire en el mediastino, no asociada a causa directa conocida. Es de infrecuente ocurrencia y su manifestación con síntomas referidos a la región cervical en forma exclusiva lo es más aún. Reportamos dos casos relacionados a actividad deportiva intensa, que se presentaron con síntomas cervicales, que no fueron diagnosticados en una primera consulta en el Servicio de Urgencia. La evaluación inicial incluyó un estudio radiológico de cuello en un caso y de columna cervical en el otro, de los cuales sólo la

Ovalle P. Neumomediastino espontáneo: Enfisema retrofaríngeo forma de presentación no habitual. Rev Chil Radiol 2005; 11: 116-121.

Correspondencia: Dr. José D. Arce V.

Clínica Santa María. Avda Santa María 0410.

E-mail:jarce@csm.cl segunda lectura en el Servicio de Radiología permitió detectar aire prevertebral. El estudio posterior con tomografía computada demostró un neumomediastino con extensión al cuello sin patología pulmonar subyacente. La evolución de los pacientes fue satisfactoria. Se discute la fisiopatología del neumomediastino y se comenta la sobre-evaluación a la que generalmente son sometidos estos pacientes.

Palabras clave: Dolor cervical, Pneumomediastino, Pneumomediastino espontáneo.

\section{Introducción}

El neumomediastino espontáneo (NE) se puede definir como la presencia de aire en el mediastino, no asociada a causa traumática, iatrogénica o enfermedad pulmonar subyacente conocida. En algunas publicaciones la definición del término resulta controversial, ya que incluyen afecciones respiratorias como neumonía, asma, bronquiolitis, infecciones virales y otras dentro de este grupo $^{(1-3)}$. Fue descrito por primera vez por Hamman, en $1939^{(4)}$, es una patología poco frecuente, benigna y autolimitada, que afecta principalmente a hombres jóvenes y mujeres embarazadas ${ }^{(2,5,6)}$.

Presentamos dos casos de NE asociado a actividad deportiva intensa, que consultaron en el servicio de urgencia por molestias referidas a la región cervical, no diagnosticados en una primera consulta aun cuando se dispuso de estudio radiológico simple de la región cervical. Discutimos además la utilidad y necesidad de los métodos diagnósticos complementarios empleados.

\section{Caso 1}

Paciente de 14 años, sin antecedentes mórbidos de importancia, que consulta en horas vespertinas en el Servicio de Urgencia en diciembre del 2002, por un cuadro de disfagia y odinofagia progresiva después de haber jugado fútbol y 
efectuado ejercicios de saltos con bicicleta, en forma intensa. Por sus molestias fue interconsultado un otorrinolaringólogo quien solicitó como parte de su evaluación una radiografía de cuello, en la que ninguna alteración fue advertida. Por su buen estado general el paciente fue derivado a su domicilio en donde la sintomatología fue disminuyendo gradualmente.

La segunda evaluación de la radiografía a la mañana siguiente, en el Servicio de Radiología detecta la presencia de una pequeña cantidad de aire en el espacio retrofaríngeo (Figura 1). Se informa al Servicio de Urgencia citándose telefónicamente al paciente, para su reevaluación, estudiándose con tomografía computada (TC) de cuello y tórax que demuestra neumomediastino con extensión hacia la región cervical y periaórtico hacia caudal (Figura 2). Este estudio demostró ausencia de patología pulmonar o neumotórax asociado.

El paciente se encontraba hemodinámicamente estable (PA: 123/61 mm de Hg, FC: 60 lat/min), afebril, sin apremio respiratorio y saturando $100 \%$. En el examen físico sólo destaca la faringe levemente congestiva, sin evidencia clínica de enfisema subcutáneo. Presenta un hemograma y PCR normales. Es hospitalizado con el diagnóstico de NE, para observación y monitoreo.

Evoluciona sin complicaciones. Se realizó un esofagograma, una fibrobroncoscopía y una endoscopía digestiva alta, que resultan normales. Una TC de tórax de control demostró regresión parcial del neumomediastino.

Después del estudio, estando el paciente en buenas condiciones generales y en ausencia de enfermedad causal fue dado de alta, con control ambulatorio estricto evolucionando asintomático y sin complicaciones.

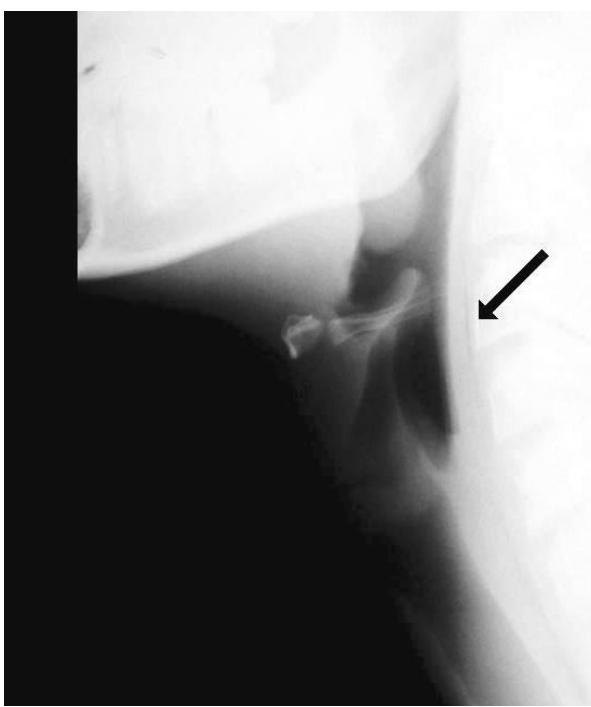

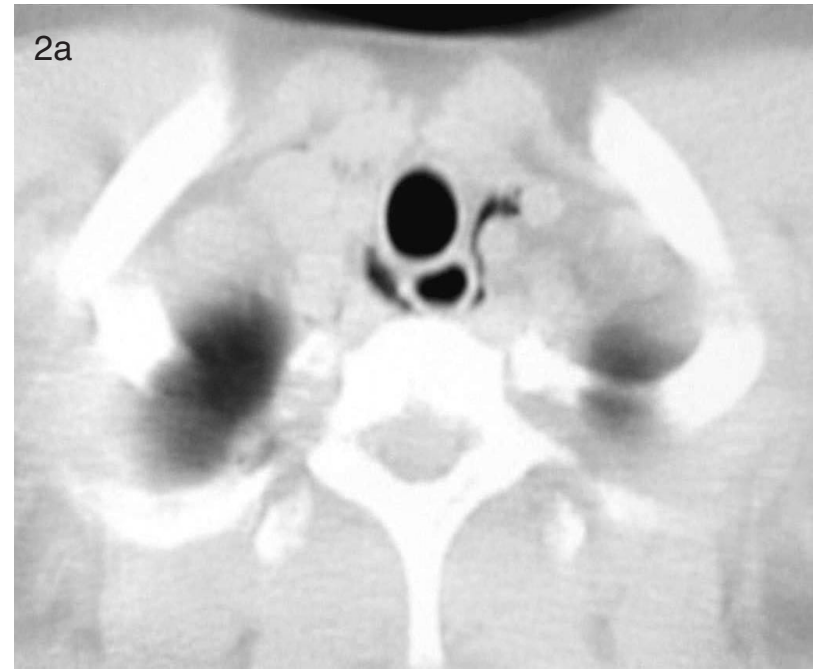
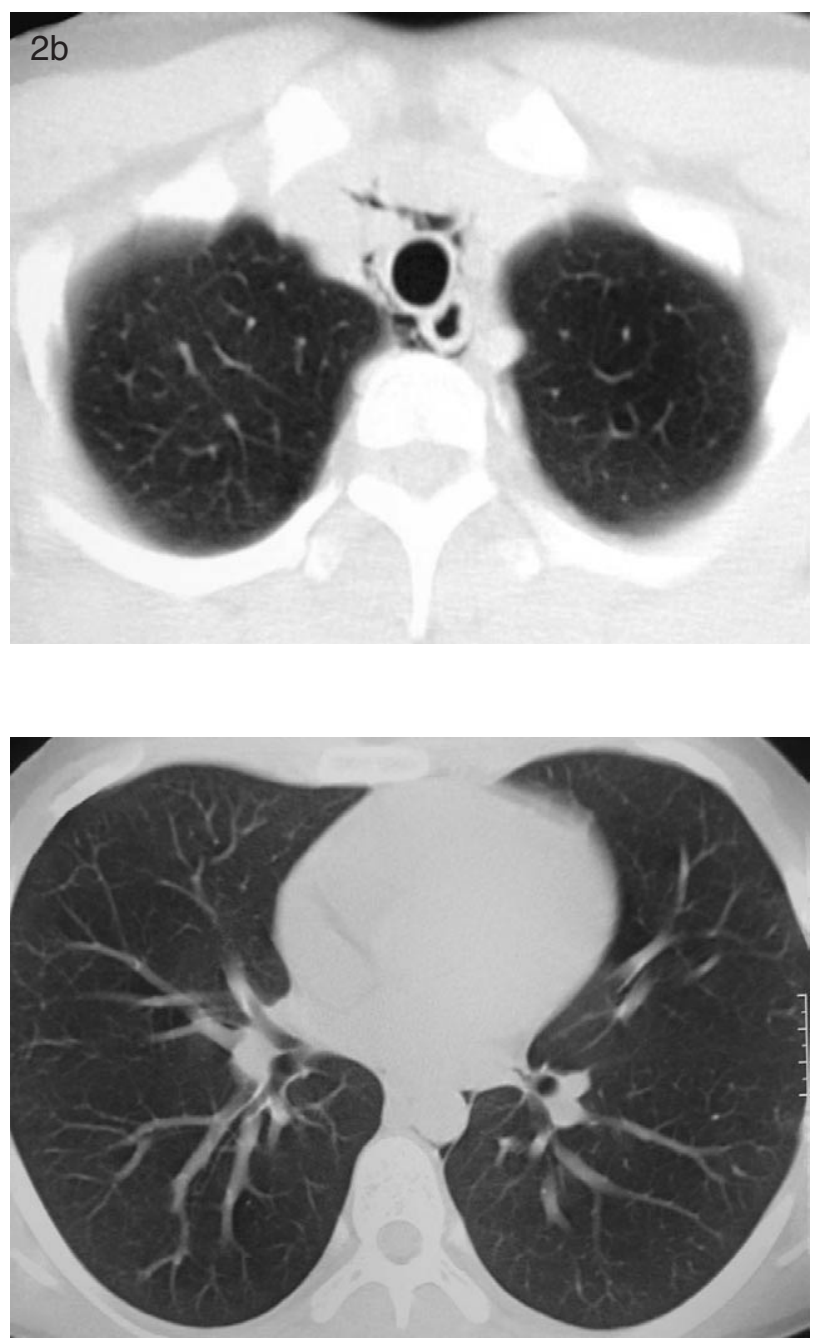

Figura 1. Radiografía simple de cuello que demuestra una fina imagen lineal radiolúcida correspondiente a aire en el espacio prevertebral (flecha). 


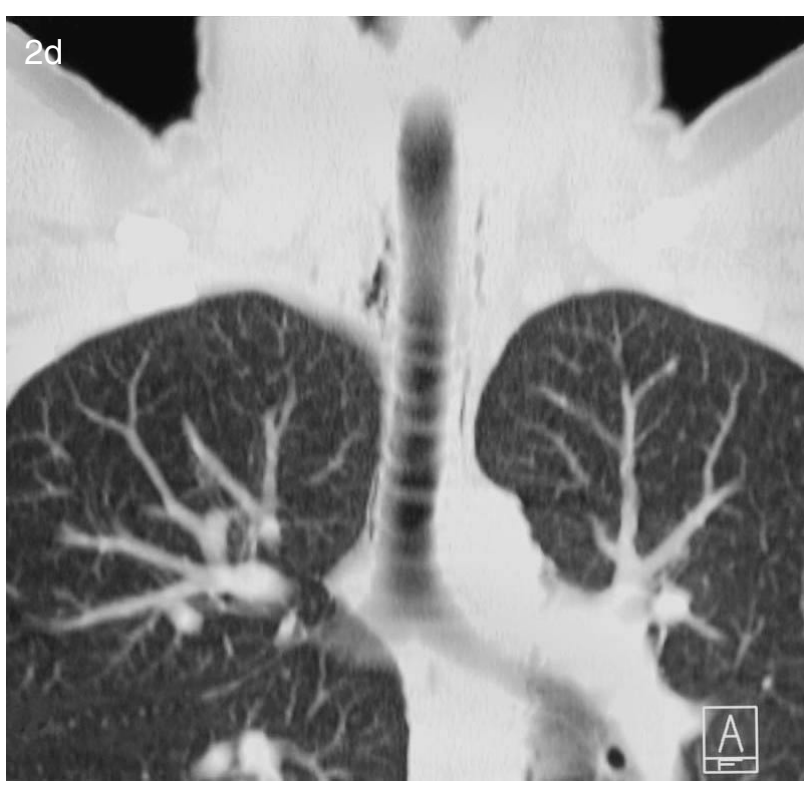

Figura 2 a-d. TC de tórax. Cortes axiales en diferentes niveles $(a, b, c)$ y reconstruccion coronal $(d)$ demostrando la presencia de aire periesofágico con extensión paravascular medial a izquierda (a) y periesofágico, perivascular y paratraqueal (c). En la recostrucción sagital (d) se observa claramente la extensión prevertebral del aire. No se observaron anomalías pulmonares ni evidencias de enfisema intersticial o neumotórax.

\section{Caso 2}

Escolar de 9 años 9 meses, eutrófico, sin antecedentes mórbidos de importancia, que en diciembre de 2004, horas después de nadar y jugar en la piscina, comienza con odinofagia, dolor cervical y retroesternal. Acude al Servicio de Urgencia, donde se solicitó evaluación por traumatólogo que indica una radiografía de columna cervical que se interpreta como normal, con ausencia de patología osteoarticular (Figura 3). Fue enviado a su domicilio con el diagnóstico de tortícolis, indicándose un collar blando y analgesia. La evolución posterior fue con disminución progresiva de su sintomatología.

La segunda lectura de las imágenes en el Servicio de Radiología, detecta aire en el espacio prevertebral, se advierte al Servicio de Urgencia que cita nuevamente al paciente para su reevaluación. Se realiza una TC de cuello y tórax que muestra neumomediastino con extensión cervical prevertebral y rodeando las estructuras vasculares del cuello. Además, se observa una pequeña cantidad de aire en relación al bronquio fuente derecho. No se detectó patología del parénquima pulmonar ni de la vía aérea o tubo digestivo superior (Figura 4).

El paciente se encontraba hemodinámicamente estable, sin enfisema subcutáneo ni apremio respiratorio. Se ingresa con el diagnóstico de NE para observación y monitoreo. Se realiza una

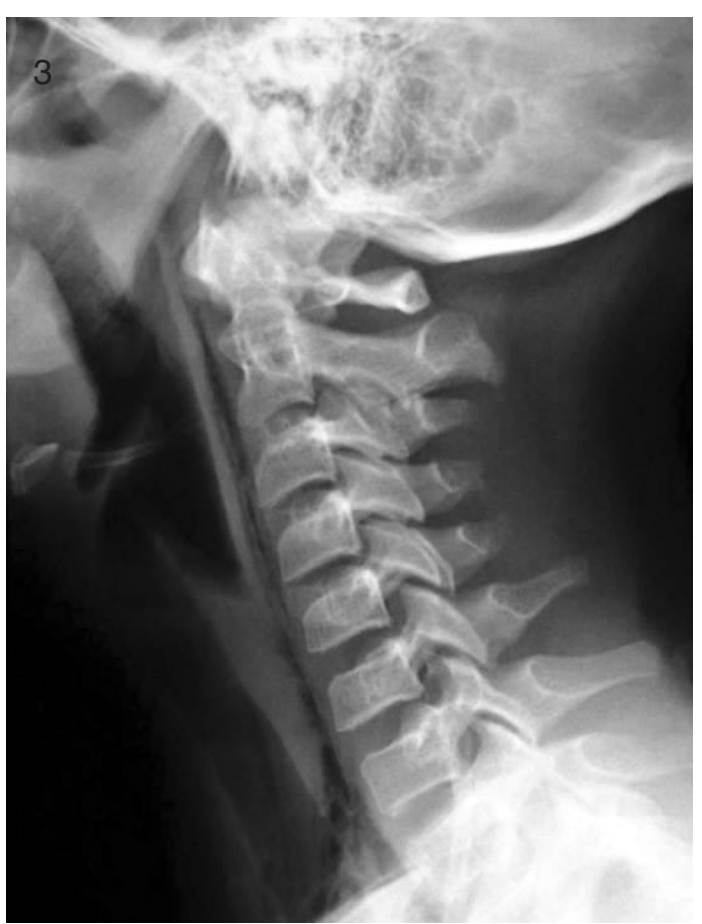

Figura 3. Radiografía simple de columna cervical proyección lateral demostrando claramente la presencia de aire prevertebral.
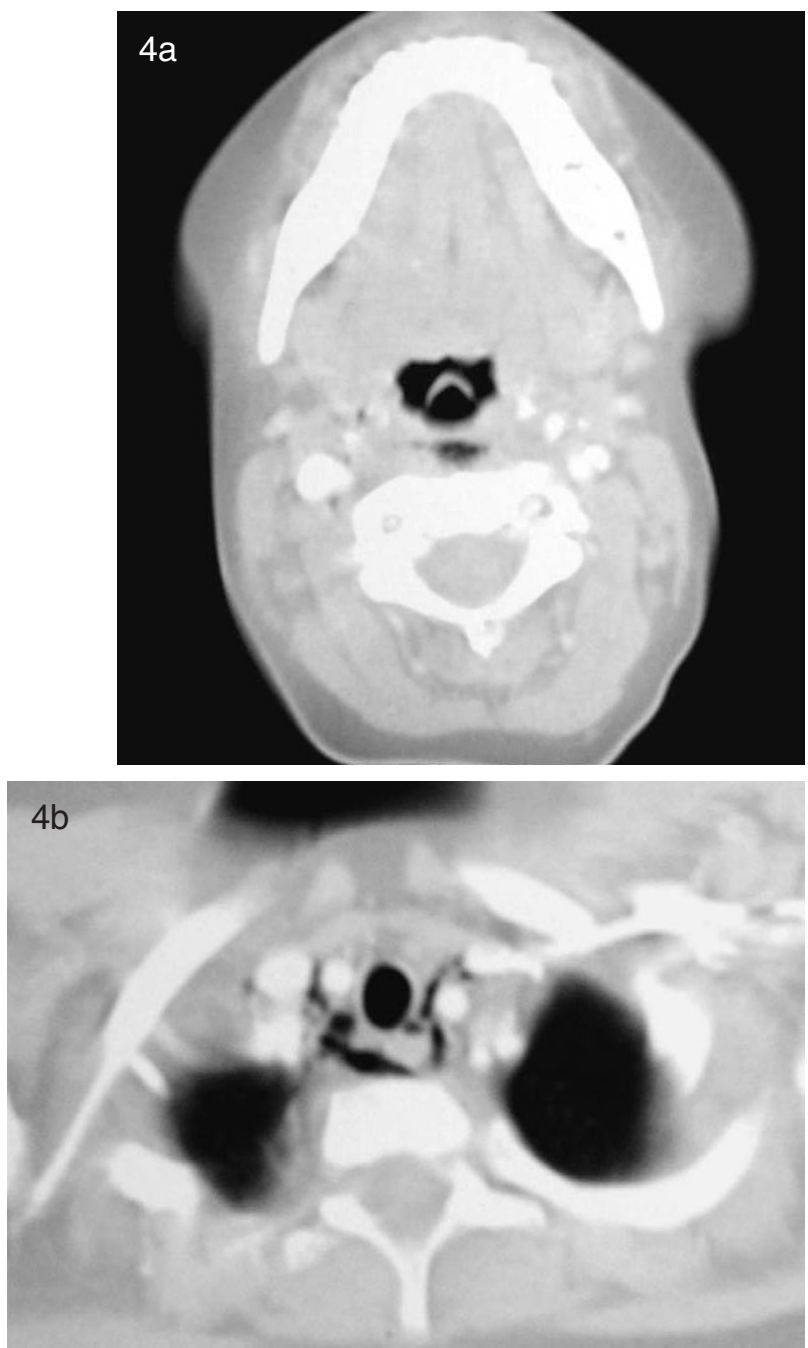

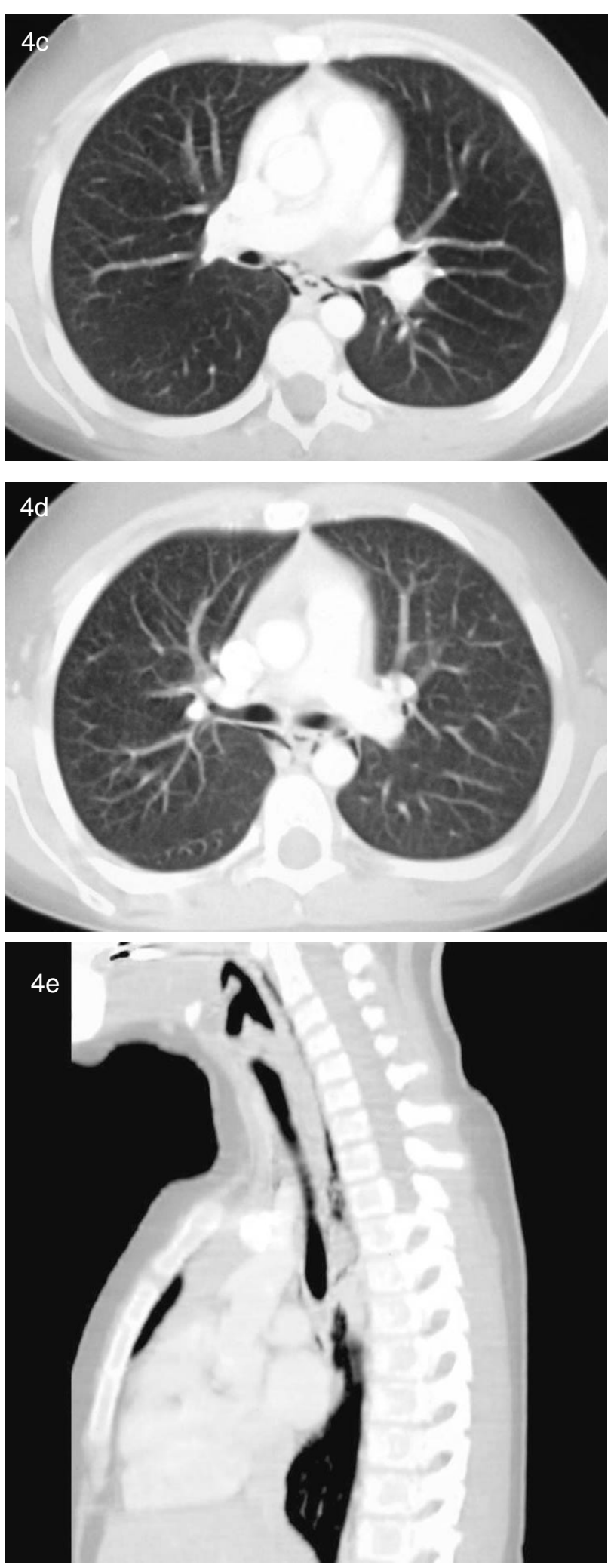

Figura 4 a-e. TC de cuello y tórax con reconstrucción sagital (e). Cortes axiales en diferentes niveles demostrando la presencia de aire periesofágico, paratraqueal, perivascular medial, alrededor del broquio fuente derecho y de la aorta descendente $(a, e)$. Nótese el aire prevertebral en la región cervical superior. No se demostró anomalías pulmonares, neumotórax, ni enfisema intersticial. fibrobroncoscopía que descarta una lesión traqueal. El niño evoluciona sin complicaciones y es dado de alta a las veinticuatro horas, con control ambulatorio presentando una evolución libre de síntomas.

En ninguno de los casos tuvimos antecedentes sobre consumo de tabaco o drogas.

\section{Discusión}

La incidencia de NE es baja, variando de 1 en 800 a 1 en 42.000 consultas a los servicios de emergencia, en las distintas series publicadas ${ }^{(5-11)}$. Una mayor incidencia ha sido reportada en hospitales militares ${ }^{(9)}$. Aunque no se conoce qué porcentaje corresponde al subgrupo asociado a actividad deportiva, existen reportes de casos en pacientes jóvenes en quienes probablemente ella esté involucrada ${ }^{(2,5)}$. Mihos y colaboradores ${ }^{(5)}$ publicaron una serie de nueve hombres y una mujer, entre $15 \mathrm{y}$ 25 años relacionados a diferentes deportes (buceo: 4 , básquetbol: 2 , fútbol: 3 , vóleibol: 1 ). Probablemente es subdiagnosticado en pacientes que presentan dolor agudo torácico o cervical en forma aislada y los reportes bajo la edad de 18 años son raros ${ }^{(2)}$. En nuestros pacientes una actividad deportiva intensa como fueron fútbol, ejercicios en bicicleta y natación está directamente relacionada con los síntomas.

El neumomediastino en general puede tener distintas etiologías como son ruptura de la vía aérea, del esófago o de una víscera hueca, barotrauma asociado a ventilación mecánica, asma, trauma, etc. ${ }^{(1,12)}$. Se debe, sin embargo, considerar que existen potenciales fuentes extratorácicas de aire que alcanzan al mediastino como lo son traumatismo de cara o cuello, extracción dental o bien desde la cavidad abdominal vía peritoneal o retroperitoneal después de la ruptura de una víscera hueca ${ }^{(12)}$.

En algunos casos, no hay un evento traumático o iatrogénico desencadenante, ni existe una enfermedad pulmonar subyacente. Esta situación es conocida como NE y, si bien la causa directa es desconocida, entre posibles mecanismos fisiopatológicos están el esfuerzo físico, tos severa, vómitos, cetoacidosis, o aspiración transitoria de cuerpo extraño.

Exista o no una causa orgánica conocida, el mecanismo en común es el aumento súbito de la presión intraalveolar, asociada a tos o espiración contra una glotis cerrada (maniobra de Valsalva) ${ }^{(5)}$. Cuando este incremento excede la presión de la vascularización pulmonar, se produce la ruptura alveolar en su base, dando salida a aire con resultado de enfisema intersticial, que diseca las vainas peribroncovasculares y los septos interlobares. Por el gradiente de presión, el aire puede progresar hacia los hilios y producir neumomediastino y neumotórax o bien puede progresar hacia la periferia pulmonar y producir bulas subpleurales y eventualmente, 
neumotórax. La ruptura de los alvéolos directamente al espacio pleural puede provocar un neumotórax, sin evidencia de enfisema intersticial o neumomediastino. Finalmente el aire puede extenderse hacia los tejidos subcutáneos de la pared torácica y del cuello generalmente hacia anterior, siguiendo los planos faciales y eventualmente hacia el canal raquídeo (2,12).

En la serie de Mihos y col., con 10 pacientes, en un rango de edad de 15-25 años, afectados por NE asociado a deportes ${ }^{(5)}$, los síntomas y signos de presentación más comunes fueron dolor retroesternal $(90 \%)$, disnea $(30 \%)$, disfagia $(20 \%)$, enfisema subcutáneo $(90 \%)$ y signo de Hamman $(90 \%)$. Este último consiste en un crujido retroesternal a la auscultación que se exacerba durante el sístole ${ }^{(4)}$. El dolor cervical y leve a moderada odinofagia fueron observados sólo en un $20 \%$ de los pacientes.

Otros síntomas y signos descritos en otras series han sido tortícolis ${ }^{(2)}$, irradiación del dolor hacia el dorso, disfonía y ausencia de la matidez cardiaca a la percusión(6). En casos más graves se ha observado cianosis y compromiso hemodinámico ${ }^{(13,14)}$. Smith y colaboradores reportaron 4 casos de NE con síntomas limitados a la cabeza y el cuello, que fueron atendidos en la primera consulta por un otorrinolaringólogo, al igual que uno de nuestros pacientes ${ }^{(15)}$.

Aun cuando la sintomatología predominante del neumomediastino es el dolor retroesternal y el enfisema subcutáneo es un signo común, ello no ocurrió en nuestros casos, en donde los síntomas de presentación fueron odinofagia, disfagia y dolor cervical: la forma menos común de presentación. Estos síntomas fueron evaluados por especialistas y confundieron a una correcta orientación diagnóstica. En ambos se solicitó radiografía de la región cervical, inicialmente interpretada como normal, probablemente por la lectura orientada a la búsqueda de hallazgos específicos o lo poco aparente del hallazgo como en el caso número 1. La segunda lectura de las radiografías en el Servicio de Radiología, norma habitual para todos los exámenes de imágenes en nuestra clínica, advirtió la presencia de aire prevertebral, por lo que la oportuna comunicación con el Servicio de Urgencia permitió reevaluar a estos pacientes confirmándose la sospecha de aire y su probable origen en el tórax.

El método diagnóstico inicial más utilizado es la radiografía de tórax, en la que se puede observar la presencia de aire disecando las vainas peribroncovasculares. El estudio puede continuarse con una TC, que ayuda a precisar la etiología del neumomediastino o excluir una causa orgánica de éste ${ }^{(5,6,13,16)}$. En nuestros casos confirmó el neumomediastino con extensión cervical y descartó una patología de base especialmente del parénquima pulmonar, objetivo en común con otros casos publicados ${ }^{(5,6,13)}$. Hay varios estudios que consideran suficiente la TC y sólo realizan un esófagograma en los pacientes con vómitos repetidos o disfagia intensa ${ }^{(2,5,13)}$.

Los signos radiológicos de neumomediastino dependerán de cuáles estructuras anatómicas delimita el aire. Una suficiente cantidad de aire permitirá delimitar el timo dando el signo clásico de «alas de ángel». También el neumoprepericardio es un hallazgo común y es bien visualizado en proyección lateral del tórax. El aire alrededor de las estructuras vasculares puede desplegar una imagen en anillo o tubular. La pared bronquial o traqueal puede ser bien visualizada en presencia de aire periférico a ella (Figuras 2 d; 4 b, d). El aire que se ubica por detrás del pericardio da el signo del diafragma continuo. En nuestros casos los pacientes fueron estudiados con TC al día siguiente de producido el neumomediastino y la cantidad de aire es escasa y delimita parcialmente las estructuras mediastínicas de los compartimentos vascular y postvascular (Figuras 2 y 4).

En nuestros pacientes, a pesar de los hallazgos, por indicación clínica se continuó la evaluación con técnicas endoscópicas. Chapledaine y colaboradores ${ }^{(1)}$ reportaron una serie de 53 casos de NE de distintas etiologías y analizaron los métodos diagnósticos empleados. El promedio de radiografías de tórax por hospitalización fue de 3 (rango: 1-16); se realizaron además cinco esófagogramas y dos TC en pacientes sin una causa clara. Todos los resultados fueron normales. Solamente tres pacientes presentaron un neumotórax durante su evolución, de los cuales ninguno requirió drenaje, concluyeron que, dado el riesgo de radiación, especialmente en niños, el diagnóstico debería hacerse con una radiografía de tórax única y los controles radiológicos deberían depender exclusivamente de una condición clínica que la justifique; el uso de la TC sería cuestionable, ya que el NE es una entidad benigna. El esofagograma debería reservarse para los casos con historia de vómitos, derrame pleural concomitante o síntomas sistémicos. Creemos, sin embargo, que una patología pulmonar o mediastínica subyacente sólo puede ser bien investigada por TC la cual debería indicarse en todos los casos, lo que está de acuerdo a algunas publicaciones ${ }^{(5)}$.

Otros grupos coinciden en que los niños con NE son sobreevaluados especialmente con estudios endoscópicos invasivos, riesgosos y la mayoría de las veces innecesarios ${ }^{(13)}$. También debe considerarse que éstos se asocian a maniobras de Valsalva que pueden incrementar el neumomediastino. 
EI NE es una condición benigna, autolimitada, que evoluciona hacia la resolución espontánea en 3 a 15 días ${ }^{(17)}$. Nuestros pacientes se mantuvieron 24 horas en observación, mientras que en otras series el promedio de días de hospitalización fluctúa entre 3,5 y 4,5 días $^{(2,5,13)}$. De todas las series revisadas, sólo en un paciente se observó una recurrencia sin secuelas posteriores. Dekel y colaboradores ${ }^{(2)}$ realizaron un seguimiento de 9 menores de 16 años con NE durante un promedio de 82,5 meses, encontrando en cinco de ellos alteraciones del VEF ${ }_{1}$ en la espirometría o desarrollo de crisis asmáticas recurrentes en los meses siguientes, hechos no observados en nuestros casos. Actualmente ya se conoce la mayor incidencia de NE en pacientes asmáticos, pero sería necesario comprobar si es también un indicador de una predisposición al desarrollo de un asma bronquial.

\section{Conclusión}

EI NE es una patología infrecuente, benigna, que puede ser provocada por la actividad deportiva. El enfisema retrofaríngeo como forma de presentación es poco común y debe ser tenido en cuenta, especialmente por especialistas, ante síntomas cervicales de presentación aguda en pacientes jóvenes. Su evolución es autolimitada y una investigación agresiva de su causa es innecesaria como asimismo las hospitalizaciones prolongadas, especialmente si se descarta una patología subyacente. Consideramos que una radiografía de tórax, como método de diagnóstico inicial y una TC para descartar patologías torácicas de base son suficientes para un manejo clínico adecuado. Dada la buena evolución clínica observada en todos los casos, el esófagograma sólo se justificaría en aquéllos con historia de vómitos, derrame pleural o síntomas sistémicos.

\section{Bibliografía}

1. Chapdelaine J, Beaunoyer $M$, Daigneault $P$, Bérubé D, Bütter A, et al. Spontaneous Pneumomediastinum: Are We Overinvestigating? J Pediatr Surg 2004; 39: 681-4.

2. Dekel B, Paret G, Szeinberg A, Vardi A, BarzilayZ.
Spontaneous pneumomediastinum in children: clinical and natural history. Eur J Pediatr 1996; 155: 695-7.

3. Damore DT, Dayan PS. Medical causes of neumomediastinum in children. Clin Pediatr 2001; 40: 87-91.

4. Hamman L. Spontaneous mediastinal emphysema. Bull Johns Hopkins Hosp 1939; 64: 1-21.

5. Mihos P, Potaris K, Gakidis I, Mazaris E, Sarras E, Kontos Z. Sports-Related Spontaneous Pneumomediastinum. Ann Thorac Surg 2004; 78: 983-6.

6. Langwieler T, Steffani K, Bogoevski, Mann O, IzbiciJ. Spontaneous Pneumomediastinum. Ann Thorac Surg 2004; 78: 711-3.

7. Abolnik I, Lossos IS, Breuer R. Spontaneous Pneumomediastinum. A report of 25 cases. Chest 1991; 100: 93-5.

8. Munsell WP. Pneumomediastinum. A report of 28 cases and review of the literature. JAMA 1967; 202: 689-93.

9. McMahon DJ. Spontaneous pneumomediastinum. Am J Surg 1976; 131: 550-1.

10. Yellin A, Lidji M, Lieberman Y. Recurrent spontaneous pneumomediastinum. The first reported case. Chest 1983; 83: 935.

11. Yellin A, Gapany-Gapanivicius M, LiebermanY. Spontaneous pneumomediastinum: is it a rare cause of chest pain? Thorax 1983; 38: 383-5.

12. Tocino I, Goodman L, Putman C. ed. Critical Care Imaging. $3^{\text {rd }}$ ed. USA: W.B. Saunders Company, 1992; 137-142.

13. Weissberg D, Weissberg D. Spontaneous mediastinal emphysema. Eur J Cardiothorac Surg 2004; 26: 885-8.

14. Nounla J, Trobs R, Bennek J et al. Idiophatic spontaneous pneumomediastinum: an uncommon emergency in children. J Pediatric Surg 2004; 39: E 23-24.

15. Smith J, Hsu J. Spontaneous pneumomediastinum presenting with retropharyngeal emphysema. Am J Otolaryngol 2004; 25: 290-4.

16. Gerazounis M, Athanassiadi K, Kalantzi N, Moustardas M. Spontaneous pneumomediastinum: a rare benign entity. J Thorac Surg 2003; 126: 774-776.

17. Chalumeau M, Clainche LL, Sayeg $\mathrm{N}$ et al. Spontaneous pneumomediastinum in children. Pediatr Pulmonol 2001; 31: 67-75. 